\title{
Nuclear factor- $\kappa B$ signaling negatively regulates high glucose-induced vascular endothelial cell damage downstream of the extracellular signal-regulated kinase/c-Jun N-terminal kinase pathway
}

\author{
YUNZHI CHEN $^{1,2}$, FANG GUO ${ }^{1}$, ZHENG RU $^{1}$, HONGRU KONG ${ }^{1}$, HONGWEI SUN $^{1}$, \\ HUAJUN YU ${ }^{1}$, WENJUN YANG ${ }^{1}$, QIYU ZHANG ${ }^{1}$ and MENGTAO ZHOU ${ }^{1}$ \\ ${ }^{1}$ Department of General Surgery, The First Affiliated Hospital of Wenzhou Medical University; \\ ${ }^{2}$ Department of Gynecology and Obstetrics, The People's Hospital of Wenzhou, Wenzhou, Zhejiang 325000, P.R. China
}

Received October 14, 2016; Accepted June 22, 2017

DOI: 10.3892/etm.2017.4999

\begin{abstract}
Diabetes mellitus (DM)-induced high blood sugar severely damages vascular endothelial cells (VECs), which are in direct contact with the blood. Diabetic complications cause difficulties in skin wound healing and VECs are important for this process. Previous studies demonstrated that high blood sugar delayed the repair of wounded VECs, but the underlying mechanism has remained elusive. To explore the effects of diabetic conditions on VEC damage, cells were incubated in a medium with high glucose and then subjected to RNA-sequencing based transcriptome analysis. The results revealed that numerous biological processes were altered by HG stress, including extracellular matrix-receptor interaction, NOD-like receptor signaling and the nuclear factor (NF)- $\mathrm{kB}$ pathway. HG treatment increased the levels of phosphory-

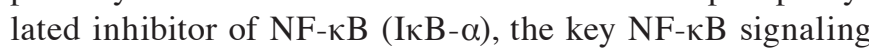
regulator as well as the transcripts of plasminogen activator inhibitor-1 and interleukin-8, two inflammatory response markers. Treatment with extracellular signal-regulated kinase (ERK)- and c-Jun N-terminal kinase (JNK)-specific inhibitors U0126 and sp600125, respectively, led to the activation

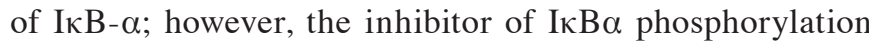
Bay11-7082 did not affect ERK and JNK activity, suggesting that ERK/JNK signaling occurs upstream of NF- $\mathrm{KB}$ in VECs. The present study provided useful information regarding the effects of diabetes on VECs, which may provide approaches
\end{abstract}

Correspondence to: Professor Mengtao Zhou, Department of General Surgery, The First Affiliated Hospital of Wenzhou Medical University, Room 403, 13 Nanbaixiang Road, Wenzhou, Zhejiang 325000, P.R. China

E-mail:wsshpcyz@126.com

Key words: nuclear factor- $\kappa \mathrm{B}$, high glucose, vascular endothelial cell, damage, extracellular signal-regulated kinase/c-Jun N-terminal kinase for therapies of diabetes-associated complications in the future.

\section{Introduction}

With exponential increases in the number of individuals affected by diabetes mellitus (DM), this disease represents a great medical and socioeconomic challenge worldwide. Despite various treatment strategies adopted, vascular complications represent a major problem causing morbidity and mortality of diabetic patients. Early in the course of diabetes, intracellular hyperglycemia causes endothelial dysfunction and endocrine disorders (1-3). The overall result is tissue dysfunction, which results in the formation of non-healing ulcers.

Skin wound repair involves a series of coordinated processes such as cell proliferation and migration, collagen deposition and remodeling, wound contraction and angiogenesis. These mechanisms involve various cell types, mostly including fibroblasts/myofibroblasts, keratinocytes and endothelial cells $(4,5)$. Due to their incapacity to regulate glucose influx, vascular endothelial cells (VEC) represent an important target for DM-induced damage. The hyperglycemia in diabetic patients has resulted in difficulties in wound healing, particularly altered angiogenesis and extracellular matrix remodeling (6), while the nature of the linkage has remained elusive. Extensive studies have described alterations in cell migration associated with diabetic conditions. For instance, Lerman et al (7) demonstrated that fibroblasts from diabetic mice migrated $75 \%$ less than those from normoglycemic mice and displayed a constant response to hypoxia, a condition commonly present in chronic wounds. Recently, an inhibition phenomenon similar to that in mice was observed in keratinocytes cultured in a high-glucose (HG) environment (8), which suggested that HG has a significant role in delaying wound repair. Mitogen-associated protein kinases (MAPKs) were identified to be involved in reactive oxygen species (ROS) production upon HG stress (9). However, these studies failed to address the detailed cellular regulatory mechanisms involved in this phenomenon. 
The present study analyzed the effects of HG on transcriptome changes in VECs via RNA-sequencing (seq) analysis. In addition, the association between $\mathrm{NF}-\kappa \mathrm{B}$ and two MAPK members, extracellular signal-regulated kinase (ERK) and c-Jun N-terminal kinase (JNK), were analyzed using pharmacological inhibitors. RNAs differentially expressed by VEGs under HG conditions and their associated biological processes were identified, and the association between NF- $\mathrm{NB}$ and MAPK pathways under diabetic conditions was analyzed.

\section{Materials and methods}

VEC culture. The EA.hy926 human endothelial cell line was obtained from the Cell Bank of the Chinese Academy of Sciences (Shanghai, China). These cells were immediately expanded and frozen so that the cell line could be restarted every 3 months. Cells were cultured in low-glucose (LG) Dulbecco's modified Eagle's medium (GE Healthcare Life Sciences, Little Chalfont, UK) supplemented with $10 \%$ fetal bovine serum (GE Healthcare Life Sciences), $100 \mu \mathrm{g} / \mathrm{ml}$ streptomycin and $100 \mathrm{U} / \mathrm{ml}$ penicillin. The cultures were maintained at $37^{\circ} \mathrm{C}$ in a humidified chamber with $5 \% \mathrm{CO}_{2}$ and $95 \%$ air, and the medium was replaced every 2 days. The cultured cells were digested and passaged with $0.25 \%$ trypsin (Gibco; Thermo Fisher Scientific, Inc., Waltham, MA, USA) after the cells reached $\sim 90 \%$ confluency. The cells were then seeded at $2.5 \times 10^{4}$ cells/well on 6 -well plates or at $1 \times 10^{4}$ cells/well on 48 -well plates and cultured for $24 \mathrm{~h}$. The EA.hy926 cells in the LG or HG group were treated with 5.5 or $35 \mathrm{mM}$ glucose, respectively, for 3 days. The cells were cultured in 6 well plates, and treated with $1 \mu \mathrm{M}$ Bay117082 (cat. no. 19542-67-7; Sigma-Aldrich; Merck KGaA, Darmstadt; Germany), U0126 (cat. no, 109511-58-2; Sigma-Aldrich; Merck KGaA), and sp600125 (cat. no. 129-56-6; Sigma-Aldrich; Merck KGaA) for $1 \mathrm{~h}$ before harvesting the cells.

RNA sequencing. Total RNA was extracted from VECs from the two groups for the RNA-seq experiments. RNA-seq experiments and data analysis were performed by Novel Bioinformatics Co., Ltd. (Shanghai, China). All of the information of altered genes was stored on a personal hard disk and is available on request.

Analysis of the pathway. Pathway analysis was performed to determine the significant pathways enriched by differentially expressed genes under $\mathrm{HG}$ according to the Kyoto Encyclopedia of Genes and Genomes (KEGG; http://www.genome.jp/kegg/), Biocarta (https://cgap.nci.nih.gov/Pathways/BioCarta_ Pathways) and Reactome (http://www.reactome.org/) databases. Fisher's exact test was performed followed by Benjamini-Hochberg multiple testing correction for isolating significantly changed pathways, and the threshold of significance was defined by the $\mathrm{P}$-value $(\mathrm{P}<0.05)$ and false-discovery rate (10).

Total RNA extraction, complementary DNA synthesis and polymerase chain reaction $(P C R)$. Total RNA was extracted from the VECs of the HG and LG groups. The cell monolayer was rinsed with ice-cold PBS. The cells then lysed by adding $1 \mathrm{ml}$ TRIzol reagent (Invitrogen; Thermo Fisher Scientific, Inc.)
Table I. Primer sequences used for polymerase chain reaction.

\begin{tabular}{ll}
\hline Primer & \multicolumn{1}{c}{ Sequence (5'-3') } \\
\hline PAI-1 F & GAGACTGAAGTCGACCTCAG \\
PAI-1 R & CTGTCCATGATGATCTCCTC \\
GAPDH F & GACCTGCCGTCTAGAAAAAC \\
GAPDH R & CTGTAGCCAAATTCGTTGTC \\
IL-8 F & GGTGCAGTTTTGCCAAGGAG \\
IL-8 R & TTCCTTGGGGTCCAGACAGA \\
Caspase-3 F & TGATGATGACATGGCGTGTC \\
Caspase-3 R & GTTGCCACCTTTCGGTTAAC \\
CCL13 F & CGTCCCATCTACTTGCTGCT \\
CCL13 R & TCAAGTCTTCAGGGTGTGAGC
\end{tabular}

F, forward; R, reverse; IL, interleukin; CCL, chemokine ligand 13; PAI-1, plasminogen activator inhibitor-1.

per each 3.5-cm diameter 6-well dish, and $0.2 \mathrm{ml}$ chloroform was added. Total RNA ( $1 \mu \mathrm{g})$ was reverse-transcribed by using a GoScript Reverse Transcription kit (A5003; Promega Corp., Madison, WI, USA) following the manufacturer's instructions. The kit included Buffer, dNTP, Oligo (dT), RNase inhibitor and reverse transcriptase. The reverse transcription reaction was subjected to the following conditions: $65^{\circ} \mathrm{C}$ for $5 \mathrm{~min}, 42^{\circ} \mathrm{C}$ for $15 \mathrm{~min}, 75^{\circ} \mathrm{C}$ for $15 \mathrm{~min}$. The SYBR-Green kit (Bio-Rad Laboratories, Inc., Hercules, CA, USA) was used for quantitative PCR assay according to the manufacturer's instructions (5 $\mu 1$ of 2X SYBR-Green buffer, $1 \mu \mathrm{l}$ of each $5 \mu \mathrm{M}$ primer and $3 \mu \mathrm{l}$ of $\mathrm{H}_{2} \mathrm{O}$ to total volume of $10 \mu \mathrm{l}$ for each PCR reaction). qPCR was performed with the following thermocycling conditions: $95^{\circ} \mathrm{C}$ for $3 \mathrm{~min}$; $40 \mathrm{cycles}$ for $95^{\circ} \mathrm{C}$ for $30 \mathrm{sec}, 58^{\circ} \mathrm{C}$ for $30 \mathrm{sec}, 72^{\circ} \mathrm{C}$ for $30 \mathrm{sec}$; and $72^{\circ} \mathrm{C}$ for $10 \mathrm{~min}$; followed by a holding temperature of $8^{\circ} \mathrm{C}$. The transcript levels were normalized against those of GAPDH using the $2^{-\Delta \Delta \mathrm{Ct}}$ method (11). The sequences of primers used for PCR are listed in Table I.

Western blot analysis. For extraction of total protein, the cells were lysed in an ice-cold lysis solution (7 M urea, $2 \mathrm{M}$ thiourea, 2\% CHAPS detergent, $40 \mathrm{mM}$ Trizma base, $40 \mathrm{mM}$ dithiothreitol, $1 \%$ protease inhibitor) and the lysates were centrifuged for $15 \mathrm{~min}$ at $15,000 \mathrm{x} \mathrm{g}$ at $4^{\circ} \mathrm{C}$. All reagents were sourced from Sigma-Aldrich. The protein concentration was measured via Nanodrop 100 (Thermo Fisher Scientific Inc.). A total of $20 \mu \mathrm{g}$ protein from each sample was separated by $10 \%$ SDS-PAGE and then electrotransferred onto Immobilon-P Transfer Membranes (Millipore, Tokyo, Japan). The membranes were incubated in $1 \mathrm{X}$ Tris-buffered saline containing 5\% skimmed milk and $0.05 \%$ Tween-20 for $1 \mathrm{~h}$ at room temperature $\left(25^{\circ} \mathrm{C}\right)$ and blotted with primary antibodies at overnight. For the primary antibodies, anti-phosphorylated inhibitor of $\mathrm{NF}-\kappa \mathrm{B}$ (p-IкB- $\alpha$ ) antibody (1:2,000 dilution; cat. no. 39A1431; Abcam, Cambridge, MA, USA), anti-IкB- $\alpha$ antibody (1:2,000 dilution; cat. no. ab7217; Abcam), anti-p-stress-activated protein kinase (SAPK)/JNK (Thr183/Tyr185) antibody (1:1,000 dilution; cat. no. 4668; Cell Signaling Technology, Inc., Danvers, MA, US), anti-SAPK/JNK (1:1,000; cat. no. 9252; Cell Signaling Technology, Inc.), anti-p-p44/42 MAPK (ERK1/2) 


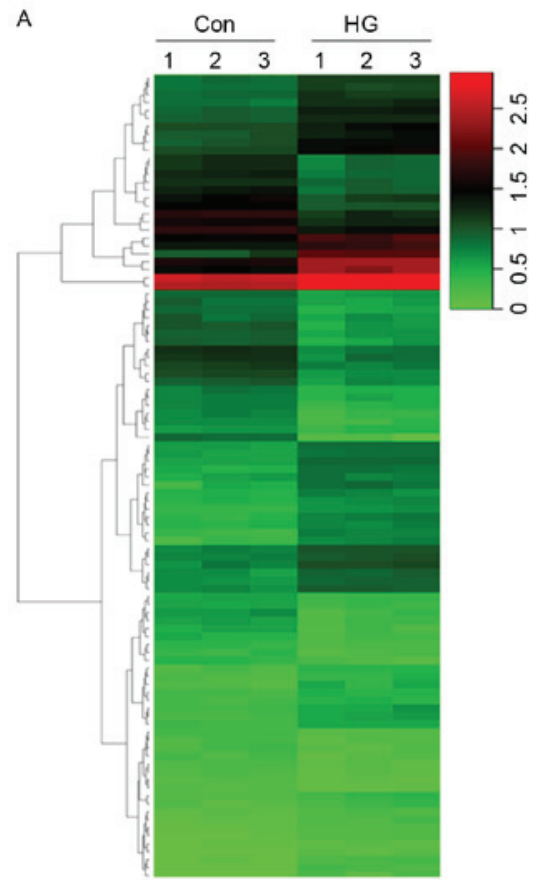

B
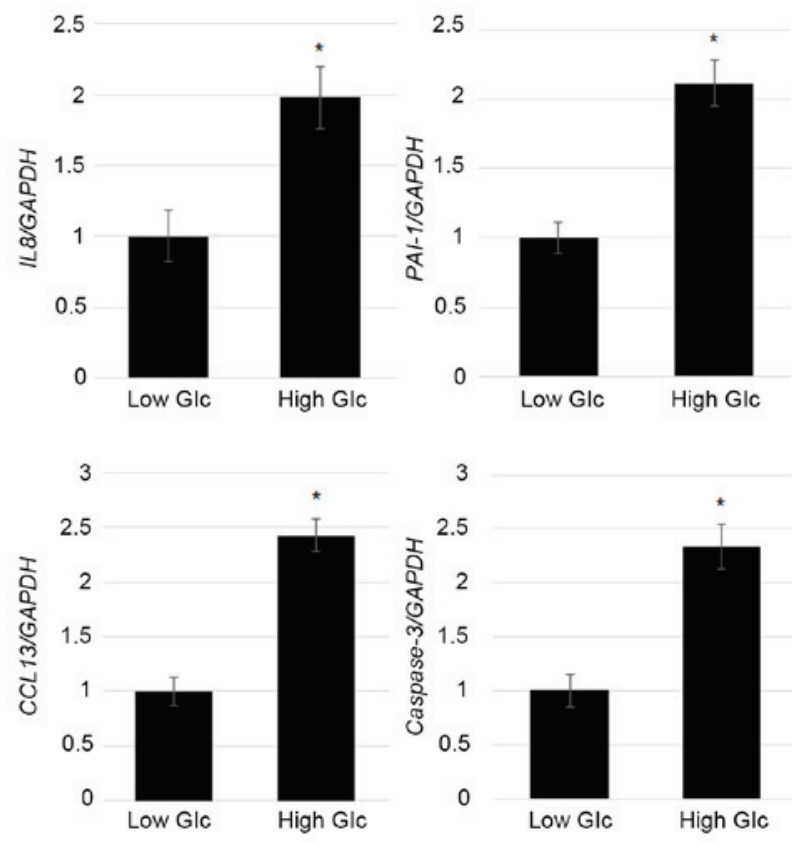

Figure 1. HG altered the transcriptome profile in VECs. (A) Heat map diagram of the genes whose expression levels in VECs were altered after HG $(35 \mathrm{mM})$ treatment for $3 \mathrm{~h}$. Gene expression is indicated by a pseudocolor scale with red denoting higher and green denoting lower gene expression levels. (B) Reverse-transcription quantitative polymerase chain reaction analysis was performed to verify the expression levels of IL-8, PAI-1, CCL13 and Caspase-3. GAPDH was used as an internal control. ${ }^{*} \mathrm{P}<0.05$ vs. Low Glc. Low Glc/Con, low glucose (5.5 mM); High Glc/HG, high glucose (35 mM); VECs, vascular endothelial cells; IL, interleukin; CCL, chemokine ligand 13; PAI-1, plasminogen activator inhibitor-1.

(Thr202/Tyr204) (1:1;000 dilution; cat. no.9101; Cell Signaling Technology, Inc.), anti-p44/42 MAPK (ERK1/2) (1:1,000 dilution; cat. no. 9102; Cell Signaling Technology, Inc.) and anti-GAPDH antibody (1:2,000 dilution; cat. no. ab8245; Abcam) were used. The membranes were incubated for $1 \mathrm{~h}$ at room temperature $\left(25^{\circ} \mathrm{C}\right)$ with an anti-mouse or anti-rabbit horseradish peroxidase-linked secondary antibody (1:2,000 dilution; cat. no. 7074; Cell Signaling Technology, Inc.). Reaction products were visualized by detection of chemiluminescence using the ECL Western Blotting Detection System (GE Healthcare Life Sciences). The images were captured on the X-ray films (Kodak; Rochester, NY, USA).

Statistical analysis. Statistical calculations were performed by using GraphPad Prism 5 software (GraphPad, Inc., La Jolla, CA, USA). All values are expressed as the mean \pm standard error. Significant differences between groups were analyzed by using the Student's t-test.

\section{Results}

Identification of HG-associated transcriptome in fibroblasts. To identify genes and pathways upregulated under HG, RNA-seq experiments were performed by using human VECs incubated under LG (5.5 mM) and HG (35 mM) conditions for $3 \mathrm{~h}$. The RNA-seq results revealed that 721 genes were differentially expressed $(>1.5$ fold-change; $\mathrm{P}<0.05)$ in the HG-treated VECs compared with those incubated under LG conditions (Fig. 1A). To verify the RNA-seq data, the HG-induced expression of inflammatory and apoptotic marker genes (IL-8, PAI-1, CCL13 and Caspase-3) were further monitored by reverse-transcription quantitative PCR. Three inflammation-associated genes (IL-8, PAI-1 and CCL13) and an apoptosis-associated gene (Caspase-3) were induced by HG stimulation (Fig. 1B), which was similar to the RNA-seq results. Furthermore, the 721 genes altered by HG stress were subjected to pathway analysis. The results demonstrated that these genes were enriched various canonical pathways, the top 15 of which are listed in Fig. 2 according to their rankings, including alcoholism as well as NF- $\kappa \mathrm{B}$ and extracellular matrix (ECM)-receptor interaction signaling pathways. NOD-like receptor signaling was identified in VEC cell with HG stress, which was previously identified as a target pathway in HG-stressed fibroblast cells (12). These data indicated that $\mathrm{HG}$ regulates a large number of genes involved in different biological processes.

Association between $N F-\kappa B$ and ERK/JNK signaling in VECs. ERK and JNK are well-known MAPKs, which are involved in diverse aspect of biology, including HG-mediated damage of VECs (9). In fibroblasts, NF- $\mathrm{B}$ signaling was identified to function upstream of JNK (13). To address the association between ERK/JNK and NF- $\kappa \mathrm{B}$ signaling, pharmacological and biochemical tests were utilized. As a key regulator, I $\mathrm{B} \alpha$ activity was analyzed after HG stress by analyzing the levels $\mathrm{p}-\mathrm{I} \kappa \mathrm{B} \alpha$ and total $\mathrm{I} \kappa \mathrm{B} \alpha$. Western blot analysis indicated HG-induced $\mathrm{p}-\mathrm{I} \kappa \mathrm{B} \alpha$ levels, particularly after $1 \mathrm{~h}$ of $\mathrm{HG}$ treatment, while total I $\kappa \mathrm{B} \alpha$ levels were not affected (Fig. 3A). In addition, the VECs were treated with the inhibitor of $\mathrm{I} \kappa \mathrm{B} \alpha$ phosphorylation Bay117082 and the p-ERK, ERK, p-JNK and JNK levels were monitored by western blot analysis. The results indicated that Bay117082 did not change ERK and JNK activities (Fig. 3B). In a different experiment, U0126 and sp600125, respective inhibitors of ERK and JNK, were applied and the 


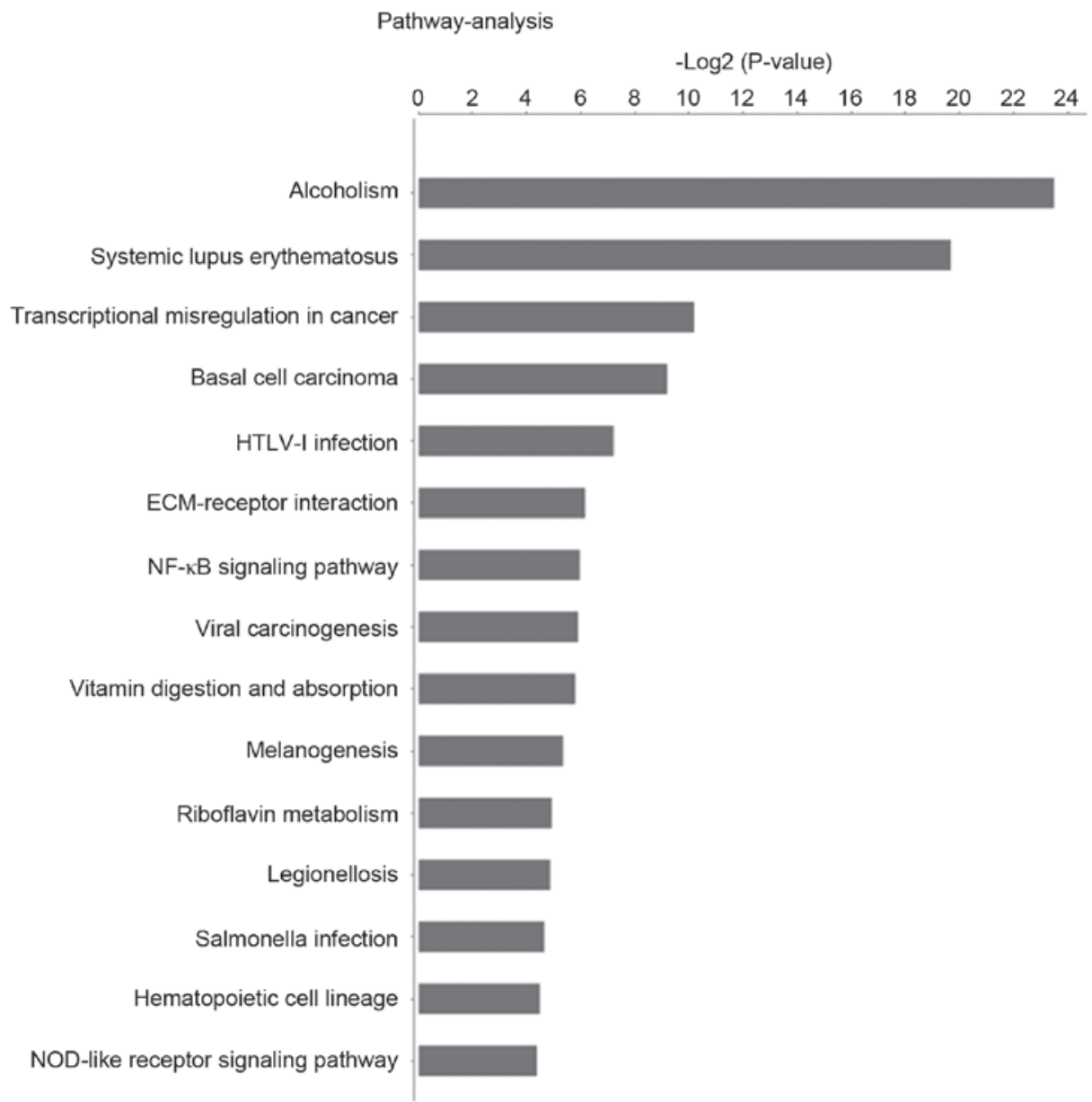

Figure 2. Top-ranking canonical pathways altered in vascular endothelial cells by high glucose stimulation. The top ranking canonical Kyoto Encyclopedia of Genes and Genomes pathways identified are listed according to P-values. The 15 pathways listed were significantly enriched $(\mathrm{P}<0.05)$, which included ECM-receptor interaction, NF- $\mathrm{kB}$ signaling, and NOD-like receptor signaling pathways. P-value, probability that the association between the more than 1.5 -fold changed genes and the canonical pathway can be accounted for by chance only. NF, nuclear factor; ECM, extracellular matrix; NOD, nucleotide-binding oligomerization domain; HTLV, human T-lymphotropic virus.

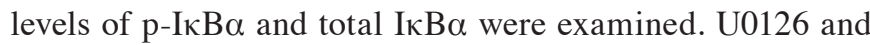
sp600125 treatment induced changes in p-ІкB $\alpha$, while IкB $\alpha$ levels were maintained (Fig. $3 \mathrm{C}$ ). These results suggested that NF- $\mathrm{KB}$ signaling functioned downstream of ERK and JNK, and that ERK and JNK negatively regulated the NF- $\mathrm{KB}$ pathway.

\section{Discussion}

VECs are the layer of blood vessels that is in direct contact with the blood, and is important for maintaining vascular tone and function, in part by the synthesis and release of certain substances in the vasculature, such as nitric oxide. VEC dysfunction contributes to the pathogenesis of vascular diseases in diabetics. While the mechanisms of VEC dysfunction in diabetes mellitus have remained to be clarified, one possibility is increased inactivation of endothelium-derived nitric oxide by ROS acting as signaling intermediates $(14,15)$. In vitro, the high ambient glucose was observed to affect endothelial and other vascular cells at the cellular level (16), delay VEC replication (17) and cause excessive cell death (18). However, the molecular mechanisms of HG-induced damage in VECs require further investigation.
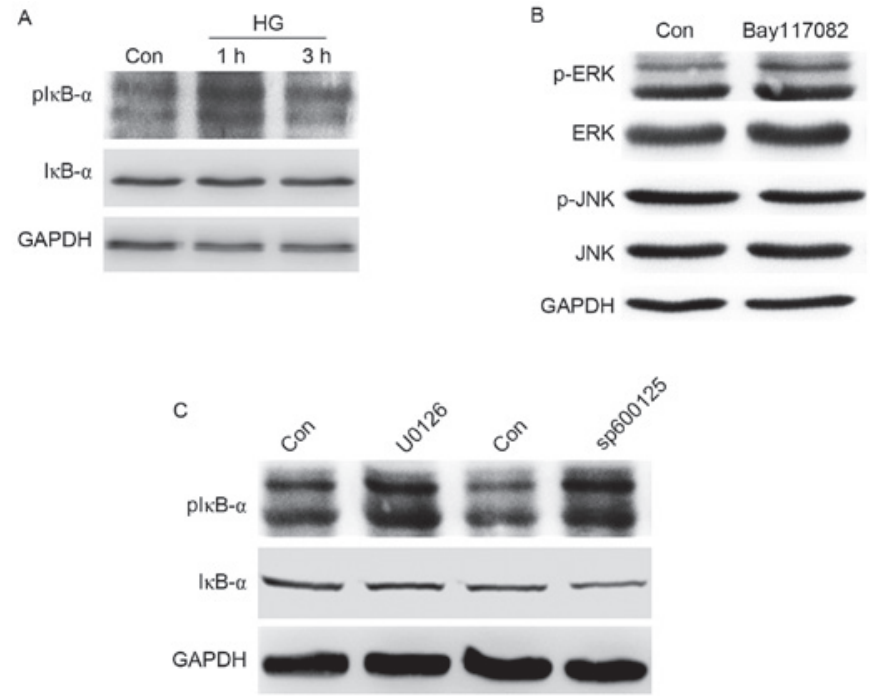

Figure 3. Association between I $\mathrm{B}-\alpha$ and JNK/ERK. (A) Western blot analysis was performed to analyze the effect of $\mathrm{HG}$ on the levels I $\mathrm{B}-\alpha$ and $\mathrm{p}-\mathrm{I} \kappa \mathrm{B}-\alpha$. (B) Effect of inhibitor of $\mathrm{I} \kappa \mathrm{B} \alpha$ phosphorylation Bay117082 on ERK/JNK and p-ERK/p-JNK levels, and (C) effects of ERK inhibitor

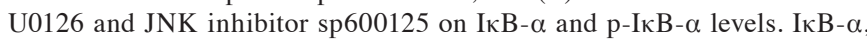
inhibitor of NF- $\kappa \mathrm{B}$; NF, nuclear factor; p-ERK, phosphorylated extracellular signal-regulated kinase; JNK, c-Jun N-terminal kinase; HG, high glucose; Con, control. 
By detecting changes in the transcriptome in VECs induced by $\mathrm{HG}, 721$ differentially expressed genes were uncovered and a possible association between the $\mathrm{NF}-\kappa \mathrm{B}$ pathway and $\mathrm{HG}$ treatment in VECs was identified. Inflammatory target genes (CCL13, PAI-1 and IL-8) associated with the activation of the $\mathrm{NF}-\kappa \mathrm{B}$ pathway were upregulated by $\mathrm{HG}$, indicating that $\mathrm{HG}$ stress activates $\mathrm{NF}-\kappa \mathrm{B}$ target genes. In addition, at the protein level, an effect of $\mathrm{HG}$ on the activation of $\mathrm{I} \kappa \mathrm{B} \alpha$, which is the most characterized and studied $\mathrm{NF}-\kappa \mathrm{B}$ regulator, was observed. In other words, $\mathrm{HG}$ activated $\mathrm{NF}-\kappa \mathrm{B}$ target genes and the activation of the $\mathrm{NF}-\kappa \mathrm{B}$ regulator-I $\kappa \mathrm{B} \alpha$ to promote the inflammatory response in VECs. PAI-1, a pro-inflammatory cytokine located downstream of NF- $\kappa \mathrm{B}$ (19), was found to be activated by HG.

It is thought that elevated levels of pro-inflammatory cytokines and proteases are present in chronic wounds due to abnormal immune responses (20). $\mathrm{NF}-\kappa \mathrm{B}$ is considered to be a regulator of the inflammatory response, which controls numerous disease states through regulating exaggerated inflammatory responses associated with certain diseases, including pulmonary diseases such as acute lung injury and acute respiratory distress syndrome (21). In Drosophila, the duration of JNK activation by lipopolysaccharide is directly controlled by the NF- $\kappa$ B protein Relish (22). The results of the present study contribute to the understanding of the molecular mechanisms regarding the interaction of $\mathrm{NF}-\kappa \mathrm{B}$ and ERK/JNK signaling in VECs under diabetic conditions. The JNK and ERK inhibitors activated I $\kappa \mathrm{B} \alpha$ as HG did. However, supplementation with NF- $\mathrm{BB}$ inhibitor Bay117082 did not affect ERK and JNK activities, implying that ERK/JNK may function upstream of $\mathrm{NF}-\kappa \mathrm{B}$ signaling in VECs. In addition, NOD-like receptor signaling was altered upon HG stress in VECs. Decreased NOD-like signaling in human fibroblast cells with $\mathrm{HG}$ stress were also observed in a previous study (12). Furthermore, genetic variation of NOD1 and NOD2 has been associated with type II DM (23), suggesting the importance of NOD-like signaling in DM-mediated stress. Previous results have suggested that ECM remodeling is important for wound repair, which is in agreement with previous findings (24). EMC receptor interaction changes induced by HG stress identified in transcriptome analysis imply that HG-mediated inhibition of wound repair in VECs may be caused by ECM and receptor interaction, subsequently resulting in signaling inhibition. The present study provided useful information for understanding the mechanisms of HG stress on VECs and the results will be important for the further study of diabetes-associated complications.

\section{Acknowledgements}

The present study was supported by a startup grant from Wenzhou Medical University (grant no. GB20140723e) and a grant from Wenzhou City Public Welfare Technology Projects (grant no. Y20140693).

\section{References}

1. Sheetz MJ and King GL: Molecular understanding of hyperglycemia's adverse effects for diabetic complications. JAMA 288 2579-2588, 2002.
2. Waltenberger J: Impaired collateral vessel development in diabetes: Potential cellular mechanisms and therapeutic implications. Cardiovas Res 49: 554-560, 2001.

3. Abaci A, Oğuzhan A, Kahraman S, Eryol NK, Unal S, Arinc H and Ergin A: Effect of diabetes mellitus on formation of coronary collateral 1vessels. Circulation 99: 2239-2242, 1999.

4. Martin P: Wound healing-aiming for perfect skin regeneration. Science 276: 75-81, 1997.

5. Gurtner GC, Werner S, Barrandon Y and Longaker MT: Wound repair and regeneration. Nature 453: 314-321, 2008.

6. Braiman-Wiksman L, Solomonik I, Spira R and Tennenbaum T: Novel insights into wound healing sequence of events. Toxicol Pathol 35: 767-779, 2007.

7. Lerman OZ, Galiano RD, Armour M, Levine JP and Gurtner GC: Cellular dysfunction in the diabetic fibroblast: Impairment in migration, vascular endothelial growth factor production, and response to hypoxia. Am J Pathol 162: 303-312, 2003.

8. Lan CC, Liu IH, Fang AH, Wen CH and Wu CS: Hyperglycaemic conditions decrease cultured keratinocyte mobility: Implications for impaired wound healing in patients with diabetes. Br J Dermatol 159: 1103-1115, 2008.

9. Zhu ZX, Cai WH, Wang T, Ye HB, Zhu YT, Chi LS, Duan YM, Sun CC, Xuan YH and Jin LT: bFGF-regulating MAPKs are involved in high glucose-mediated ROS production and delay of vascular endothelial cell migration. PLoS One 10: e0144495, 2015.

10. Draghici S, Khatri P, Tarca AL, Amin K, Done A, Voichita C, Georgescu C and Romero R: A systems biology approach for pathway level analysis. Genome Res 17: 1537-1545, 2007.

11. Livak KJ and Schmittgen TD: Analysis of relative gene expression data using real-time quantitative PCR and the 2(-Delta Delta $\mathrm{C}(\mathrm{T})$ ) method. Methods 25: 402-408, 2001.

12. Pang L, Wang Y, Zheng M, Wang Q, Lin H, Zhang $\mathrm{L}$ and Wu L: Transcriptomic study of high-glucose effects on human skin fibroblast cells. Mol Med Rep 13: 2627-2634, 2016

13. Xuan Y, Chi L, Tian H, Cai W, Sun C, Wang T, Zhou X, Shao M, Zhu Y, Niu C, et al: The activation of the NF-kB-JNK pathway is independent of the PI3K-Rac1-JNK pathway involved in the bFGF-regulated human fibroblast cell migration. J Dermatol Sci 82: 28-37, 2016.

14. Tesfamariam B and Cohen RA: Free radicals mediate endothelial cell dysfunction caused by elevated glucose. Am J Physiol 263: H321-H326, 1992.

15. Langenstroer P and Pieper GM: Regulation of spontaneous EDRF release in diabetic rat aorta by oxygen free radicals. Am J Physiol 263: H257-H265, 1992.

16. Lorenzi M and Cagliero E: Pathobiology of endothelial and other vascular cells in diabetes mellitus. Call for data. Diabetes 40: 653-659. 1991.

17. Ono H, Umeda F, Inoguchi $\mathrm{T}$ and Ibayashi $\mathrm{H}$ : Glucose inhibits prostacyclin production by cultured aortic endothelial cells. Thromb Haemost 60: 174-177, 1988.

18. Lorenzi M, Cagliero E and Toledo S: Glucose toxicity for human endothelial cells in culture. Delayed replication, disturbed cell cycle, and accelerated death. Diabetes 34: 621-627, 1985.

19. Ferran C, Millan MT, Csizmadia V, Cooper JT, Brostjan C, Bach FH and Winkler H: Inhibition of NF-kappa B by pyrrolidine dithiocarbamate blocks endothelial cell activation. Biochem Biophys Res Commun 214: 212-223, 1995.

20. Wolcott RD, Rhoads DD and Dowd SE: Biofilms and chronic wound inflammation. J Wound Care 17: 333-341, 2008.

21. Rahman A and Fazal F: Blocking NF- $\mathrm{BB}$ : An inflammatory issue. Proc Am Thorac Soc 8: 497-503, 2011.

22. Park JM, Brady H, Ruocco MG, Sun H, Williams D, Lee SJ, Kato T Jr, Richards N, Chan K, Mercurio F, et al: Targeting of TAK1 by the NF-kappaB protein Relish regulates the JNK-mediated immune response in drosophila. Genes Dev 18: 584-594, 2004.

23. Ozbayer C, Kurt H, Kebapci MN, Gunes HV, Colak E and Degirmenci I: Effects of genetic variations in the genes encoding NOD1 and NOD2 on type 2 diabetes mellitus and insulin resistance. J Clin Pharm Ther 42: 98-102, 2017.

24. Brem H and Tomic-Canic M: Cellular and molecular basis ofwound healing in diabetes. J Clin Invest 117: 1219-1222, 2007. 